Classification

Physics Abstracts

$01.65-07.60 \mathrm{~L}-07.65 \mathrm{G}$

\title{
Pierre Jacquinot and the beginnings of Fourier transform spectrometry
}

\author{
Pierre Connes \\ Service d'Aéronomie du CNRS, Verrières-le-Buisson, 91371 France
}

(Received 17 October 1991, accepted 26 November 1991)

\begin{abstract}
Résumé. - Compte rendu historique des premiers développements de la spectrométrie de Fourier au Laboratoire Aimé Cotton, sous la direction de Pierre Jacquinot, pendant les années 1954-1963.
\end{abstract}

\begin{abstract}
Historical account of the early development of Fourier Transform Spectrometry at Laboratoire Aimé Cotton under Pierre Jacquinot's leadership, over the years 1954-1963.
\end{abstract}

The most fitting tribute to Pierre Jacquinot I can think of, is an account of one line of work done under his leadership, roughly between years 1954-1963. The goal was to develop a fascinating but somewhat unlikely proposal he had just made: later-so-called Fourier Transform Spectrometry. Of course, much deeper physics went on in some other dark corners of Laboratoire Aimé Cotton; these I am not qualified to discuss. Later aspects of the same development will be found in a second paper, within an issue of Spectrochemica Acta [1] devoted to «High resolution Fourier transform spectrometry in France ». During this second period, Jacquinot had left the direction of Laboratoire Aimé Cotton (LAC) for that of Centre National de la Recherche Scientifique; but the central idea and overall impulse remained unquestionably his own.

At the 1983 Durham FTS meeting, Jacquinot evoked in his own words « the rather long train of thoughts » which led him to propose «the method now known as FTS ». In the same Proceedings [2] Peter Fellgett writes about his parallel and somewhat earlier (but then little known) development of similar ideas. These two independent contributions are today widely acknowledged as both essential to the ultimate success of FTS. Reminiscent papers by the other early workers in the field, J. Strong, H. Gebbie and L. Mertz describe their first efforts, and I contributed a study of the pionneering work by Michelson and Rubens (see also [3]). The present paper deals only with the actual doings within LAC and no attempt is made at giving a comprehensive history of FTS. Neither is this a proper review of the LAC work; the accent will be put on ideas, devices or episodes not so far deemed worth describing in print, and I apologize for their perhaps too personal character.

Both J. Connes and myself entered LAC as beginners in 1954. What kind of scenery were we provided with? The laboratory was located at Bellevue, in picturesque but somewhat 
unhandy quarters : a famous former dancing school, not exactly improved by CNRS scienceminded additions. The fairly efficient workshop was equipped with war-reparation German machine-tools ; facilities for doing electronics were primitive. Most of the work dealt with atomic spectroscopy, with emphasis on the higher-resolution aspects such as Zeeman patterns, isotope shifts and hyperfine structures. The main available tools were the large vertical-well liquid-prism spectrograph, built by $\mathrm{A}$. Couder at Aimé Cotton's request, under the celebrated 100-ton Bellevue magnet, and several recording Fabry-Perot spectrometers recently developed by Jacquinot and his pupil $\mathrm{Ch}$. Dufour. These were increasingly used for high-resolution work and $\mathrm{R}$. Chabbal was building ever more powerful compound systems. $\mathrm{P}$. Giacomo and S. Roizen produced, among other things, better FP coatings than were available anywhere else. J. Blaise and $\mathrm{H}$. Chantrel were busy full time recording and analyzing FP spectra. S. Gerstenkorn was already dreaming about isotope separation by optical means. L. Couture was studying Zeeman patterns of crystals, and B. Dossier-Roizen perfecting apodization. From all of them we learned the ways and means of scientific research.

At coffee time everybody would meet in the cramped and friendly quarters of Giacomo's office, the walls of which were decorated by his delightful cartoons. Talk was lively and ideas in constant flux ; many new ones were conceived at this so-called café-club, which Jacquinot often attended. Somewhat apart stood D. Jackson, whose larger-than-life person and doings [4] cannot be properly described in a few words. It says much for LAC that he had elected to pursue his work there. Sharing a lab with him, and listening (mostly in the dark, during long FP exposures on his superb privately-owned Hilger spectrograph) to his spectroscopic or wartime activities has been an unforgettable experience.

Required reading for newcomers was the preprint of a paper comparing from basic principles «the luminosity of prisms, gratings and Fabry-Perot étalons » [5]. Here we see a distinctive Jacquinot trademark, and one that has provided the central guideline throughout the entire development : the will and ability to treat menial instrumental matters as parts of physics just like more high-flown subjects. Unknown to the LAC group, P. Fellgett was working at the time not only on a parallel line, but from a very similar philosophy ; one could not, at this point, find better words than his own «... it is found that the phenomena which arise in a scientific instrument are just as fascinating, just as challenging and just as worthy of intellectual study, as the phenomena the instrument was intended to elucidate » [2]. That very same attitude was being drilled into the LAC students, and one hopes they are all as grateful as $\mathrm{I}$ am for the training.

At the café-club, some local wit pinned-up a topical motto : "Les petits Fabry-Perots font les grandes lumières $»$. As it was copied from an ubiquitous advertisement for electric bulbs, there was nothing modest about the message : the good old Fabry-Perot, as refurbished by Jacquinot, was fated to illuminate the world.

My first scientific assignment was shelving the powerful but unwieldy Couder prism, and replacing it by a just-available Bausch \& Lomb grating. And it proved a full success : I broke neither. Little did we guess that the second task was to keep us busy for a somewhat longer time : studying a just-published paper «Quelques perspectives d'avenir en spectroscopie instrumentale » [6], in which the essence of the FTS technique, and the basic advantages were already treated (a summary of the arguments is now available, in English, in [2]). We were also given to chew upon the somewhat cryptic 30-lines abstract of an oral communication made by $P$. Fellgett at the 1952 Columbus symposium on molecular spectroscopy about «Multichannel Spectrometry », so far the sole outwardly-visible sign of his activity. However, Fourier's name was not mentioned, so we were not even quite sure that the basic idea was the same one we were working upon. No practical details were given either, nor any reference to the Fellgett Cambridge $\mathrm{Ph}$. D. ; this is most unfortunate, because the mere knowledge that 
the author had already produced digital spectra might have put us on the right track much earlier. Since the given address was Lick Observatory, we were not, at first, aware of Fellgett's relative proximity.

Very soon, the task of developing FTS was given to J. Connes as her Ph. D. subject (I was still miserably bound to teaching physics in high-school). The goal and at least some of the limitations were clear from the onset. Here was a potentially very powerful idea, but the difficulties seemed equally formidable because we thought only in terms of the current LAC problems. Hence success implied beating large grating spectrographs and FP interferometers at their own games; any lesser goal would simply not do. The FP was already providing unlimited resolving power, but it was sadly restricted in spectral range. Rowland-circle grating spectrographs, or crossed-dispersion échelles, gave very wide range with quite decent resolution. These were the openly avowed enemies.

Which friends could we rely upon? We knew that Michelson's interferometer and fringe visibility technique also provided unlimited resolution, but had remained stuck with ambiguous reconstructions of very simple patterns; sad to confess, Rubens was then totally forgotten [3]. We had full confidence in the Jacquinot theory: our spectral range would ultimately be unlimited, with no ambiguity whatsoever; but how the deuce were we in practice ever going to perform that appalling Fourier transformation ? Thoughts ran solely on analog reconstruction. From Jacquinot's past work in acoustics a first line of approach (already mentioned in [6]) was apparent. The interferogram could be recorded on magnetic wire (these were the days before tape); then a standard wave analyzer might do the trick provided the tunable resonator $\mathrm{Q}$ was at least equal to the interferometer resolving power, the recording/playback velocity stable enough, and the dynamic range adequate... The question marks loomed very big, again considering the hoped-for resolution and range. As we shall see, an earnest attempt was to be made during a later period.

We toyed with another possibility. From P. M. Duffieux's (and B. Dossier-Roizen's) work, we knew of course that Fourier transforms were involved in diffraction theory. Hence, plans were made for recording interferograms on a photographic plate, from an intensitymodulated source through a moving slit; after developing and fixing, the plate was to be illuminated by a mercury line beam. The Fraunhofer pattern would provide some kind of answer. There was nothing absurd about the method (much later, it has been well used in speckle interferometry) but it did not look appealing: here was FTS, supposed to do overwhelmingly better than spectrographs, and we did not relish having to reintroduce the well-known idiosyncrasies of the photographic plate from the start. At best the spectra, would be swarming with artifacts.. By the time, we were able to produce half-decent interferograms, the idea had been dropped.

Interferometers we did build, plenty of them. LAC had fair experience with pressurescanned FP étalons, and the philosophy had been so far : leave well alone, and for Heaven's sake, don't attempt to move anything. Confronted with FTS, Zeno himself would have confessed the need of motion. A disemboweled old microphotometer was used to translate a mirror in our first attempt at a variable-path Michelson interferometer. Results were dismaying. Fortunately, Jacquinot, just disembarking from an OSA meeting, whisked out of his pocket some tiny cube-corner prisms (he had smuggled them in) plus a paper by E. Peck [7] describing their use ; these saved the day. We did not know Fellgett was at the time doing exactly the same. More interferometers were built (mostly of wood, except for the optics), and used with Chabbal to demonstrate all types of two-beam fringes during Jacquinot's regular Sorbonne lectures. These soon were developed commercially through an enjoyable collaboration with P. Stehlé who built excellent small Michelsons for University use. First exhibited at the Société de Physique annual show, they were to prove popular with physics departments. 
Dancers had long vanished from the Bellevue halls, and been replaced by less discrete neighbours: Diesel marine engines, which were undergoing commendably thorough tests next door to the optics lab. An environment not conducive to interferometry? Just the opposite, and one may recommend the experience to beginners ; of course we piled up truck inner tubes and heavy steel plates, and worked late at night, or Sundays. Partly as a consequence, the next interferometers were quite crazy : at some point we managed to get convinced that elimination of flexure was the key to all stability problems. The result was a Forth-bridge-like monster, all bristling with girders, with the optics in the neutral fiber plane ! Stable it may have been, but one had better wear a helmet to do any adjustment. Another one relied on huge mercury pools for supporting mirror carriage weight. All smooth, shiny and so pleasant to handle; we wallowed delightedly in the stuff for months. Today, any safety inspector would get a heart attack at the sight; we never noticed any ill effects. From this whole period, the lone positive outcome was the first LAC field-compensated interferometer for FTS [10], derived from a problème d'agrégation given by A. Maréchal.

As to spectra, we never produced a single one, and during occasional flashes of good sense, we despaired of our too-difficult goal. One was responsible for the SISAM gratinginterferometer [9] (a non-Fourier device), developed as a mere stop-gap to be pushed only for as long as FTS did not reach high resolution. Another consequence was a standard application of the scientist's Golden Rule : if your experiment declines to work, polish up the theory instead. A fair amount of mathematical work was done by J. Connes, not to be published for years, but destined to become useful later on. The reason we did not get spectra is that we were sadly innocent of digital techniques, our greatest single failure in those early times. Fourier's integrals we took as elegant devices for describing a host of physical processes ; it never entered our heads that in actual practice they could be boiled down to a set of lowly multiplications and additions... It was only in 1956 that we discovered from Dufour, who was paying a brief visit to $\mathrm{LAC}$, the mere existence of newfangled contraptions called calculateurs which he thought (he was not quite sure) might do Fourier transforms at a pinch. Still, we did not know where to get one, nor would have been capable to use it if we had. Before we could proceed any further, the blow was to fell in the shape of a paper by $\mathrm{H}$. Gebbie and G. Vanasse [10], in which low-resolution but highly-promising FIR spectra had been produced, with the help of an IBM 650 .

The time had clearly come for a meeting, and Jacquinot soon sent out invitations to a CNRS-funded «Colloque international sur les progrès récents en spectroscopie interférentielle ", to be held at Bellevue in 1957. Altogether, FTS was discussed only in a few of the talks, but it was the major novelty. The task of opening the show was given to Fellgett, who presented his interferometer (prototype of many later ones), and digital spectra, computed with Lipsom-Beevers strips. Gebbie showed his IBM-computed ones, Strong and Vanasse their FIR lamellar grating and analog computer, Mertz his fast-scanned stellar interferograms, and J. Connes exposed her budding theory. Jacquinot discussed and compared all techniques. Everything was published by Journal de Physique [9] ; according to the rules of the time, the text had to be wholly in French, and I plead guilty for the FTS translations. Ideas were pooled and personal relations established. Fellgett was to take a keen interest in LAC work, and to give some good advice now and then. For all those involved, the time of the meeting seems to have been a turning point.

It was also a turning point for another reason. All of a sudden, IBM-France organized FORTRAN courses, and gave out a few hours of free computing time on a 704, the latest and fanciest of its line. J. Connes instantly took the plunge; alas! like many another promising young physicist, she never surfaced again. Working conditions were a distinct change from Bellevue. The computer proudly stood as the central showpiece at the IBM headquarter on 
Place Vendôme, right behind huge plate-glass windows. Parisians and tourists alike gaped open-mouthed at Fourier in action, in between visits to nearby jewellers shops. The FTS mathematical study swelled up to include sampling, SNR etc.

A Canadian visitor to LAC, H. Gush, brought over a new problem in which he was an expert : near-infrared night-sky emission. This was an excellent subject because the best performance of grating spectrometers on such a low-brightness source had been very poor : a resolving power of a hundred at best. Doing ten times better looked useful, and just feasible with the 704. Moreover, both the (later so-called) Fellgett and Jacquinot advantages would play fully. One more interferometer was built, by $\mathrm{H}$. Gush and J. Connes, with a maximum displacement of a few $\mathrm{mm}$. Since the fashion had gone from wood to concrete in those days, the end product, while quite small, required two or three people to handle it. Everything was packed into the trunk of the long-suffering family car; the expedition left for Observatoire de Haute Provence, with the headlights pointing to the stars, giving them fair warning of the onslaught to come later...

The interferograms were recorded on paper, together with fringes from the green $\mathrm{Hg}$ line, and up to 6000 samples measured by eye, hand, ruler and pencil ! At the very best, getting any check on the results took a few days, but they had been worth waiting for. The night-sky spectra [11] were a success; resolving power went up to about 2000 . Next, in collaboration with $O$. Parodi, germanium-emission spectra were produced at Bellevue with the same interferometer. When the J. Connes thesis [12] was finally submitted in 1960, it contained not only the mostly-remembered theoretical part, but two demonstrative spectroscopic results.

Three newcomers came to rejuvenate the work force. The first was G. Michel, a young engineer, who organized almost from scratch the sorely-needed LAC electronics group. From then on it grew at the same pace as the needs of FTS; the benefits were reciprocal. Michel was to be an essential coauthor on most of the key papers. The second was P. Bouchareine, then an Ecole Normale Supérieure student, and a born optician. He built for his Diplôme d'Etudes supérieures (a one-year undertaking) a new and simpler form of field-widened interferometer [13]. We intended it for more studies of the night sky, but never managed to do so after he had left (others groups did, with excellent results). Third came J. Pinard, «third-cycle" student, and a born electronician; only the FTS part of his considerable activity will be mentioned here.

With such help, it will seem strange that no more spectra were to be produced after the night-sky and germanium ones for a long time. Most unfortunately J. Connes had to leave LAC for two years as maître de conférences at Caen University; this accounts in part for the slow-down in the program. Even worse, much work remained to be done on technological matters, because a solution to the specific LAC problems was by no means in sight. We wanted to get at one shot all the hyperfine structures in the atomic spectrum of a given element across the entire spectral range of a given detector, say a $\mathrm{PbS}$ cell ; this required roughly one million spectral samples, and as many interferogram ones. A back-of-theenvelope calculation immediately gave dozens of years for the 704 computing time (see [1]). Moreover, the disastrous artifacts created by path-difference and/or intensity errors had by then been fully estimated. Accuracy improvements by orders of magnitudes would be needed compared to the night-sky interferometer, particularly for extension to the visible and UV ranges.

The two main difficulties were both tackled, with unequal success. A special-purpose hybrid computer was built by G. Michel. The idea came from Jacquinot's wave-analyzer proposal. The interferogram intensity was recorded on magnetic tape in analog form; by using frequency modulation of a carrier, adequate linearity was achieved. Mercury-line fringes went to a separate track. During playback the corresponding fringe frequency could be 
demultiplied at will by digital operations. The tape was formed into a loop, and a few spectral elements were measured by cross-correlation for each turn. The first demultiplier used a forest of tubes; in winter it provided a welcome addition to the antiquated Bellevue heating system. A second version was fully transistorized. The electronics worked all right [14], but the recorder gave much trouble, particularly from tape wear. Most probably, the system would have proved usable in the end, but the whole effort collapsed when FFT's became available.

The second problem was to keep the group busy up to 1969 ; only the early efforts are described here. Out of the various possibilities discussed in [12], the step-and-integrate technique was selected. At the cost of greater complexity, it promised better accuracy, plus the rock-bottom Shannon-theorem number of samples, an essential factor at the time. The first interferometrically-controlled stepping drive, built by Pinard, is described in his thirdcycle thesis [15]. The accuracy tests were convincing, and the same technique was soon applied to the Jobin-Yvon grating engine. Interferograms were beautiful to look at. We felt we had the complete solution. The logical next step was : get spectra.

It was not to be taken at LAC. Early in 1963, as we were fiddling with the controls of our first and temperamental optical maser, a phone call came from across the Atlantic. The sender was L. Kaplan, an atmospheric physicist at Jet Propulsion Laboratory in Pasadena. He had been impressed by the night sky spectra, and was proposing a new problem : planetary NIR spectroscopy, plus unlimited use of the JPL huge battery of computers. Consulted, Fellgett gave a favourable answer. The combination proved irresistible, and six months later the Connes family left for California. This time, the luggage contained no interferometer, only schematics for the stepping drive.

The present narration may come to a halt at this point, both for the sake of suspense (next episode in [1]) and of historical logic. Shortly before, Chabbal had taken over the direction of LAC from Jacquinot, who became the new head of CNRS. While his interest remained acute, he was no longer able to provide the day-to-day on-the-spot criticism and/or encouragement we had received during one fruitful decade. Soon after, LAC moved out to luxurious new quarters at Orsay. There was a strong infusion of young blood, and some old-timers left for good. The café-club was deserted.

Let us apply in fine a heavyish dose of hindsight wisdom. Two thoughts emerge. First, it is clear that the Jacquinot overambitious initial goals proved both a short-term bane and a longterm blessing. While LAC was just toying with fancy interferometers, outsiders were churning out fine spectra. However, it was later to make up for lost time and extend the FTS range beyond the dreams of other groups.

Second, a more fundamental one. The original Fellgett-Jacquinot proposals did not arise from some out-of-the-blue technical development : neither of them at first thought in terms of electronic computing. However, both applied proper reasoning to the physics hidden behind low-light-level spectrometry, as seen from the teachings of quantum theory. This is the reason for the FTS enduring success. The technique has now weathered two major storms. The first came from nothing less than the laser revolution : laser spectroscopy has quickly gained a wide field of its own, but shows no sign of elbowing out FTS. Indeed, there are many cases of fruitful collaboration between the two. The second arose from CCD's etc. Simplistic arguments once fashionable «proved " that multiple-pixel detectors, when available, would kill FTS, but nothing of the kind has taken place. The point inherent in multiplexing a single detector remains the same ; furthermore, CCD's offer a novel way of using the solid-angle (or so-called Jacquinot) advantage, and imaging FT spectrometers are getting popular. Lastly, FTS has at least shown the way for two other major developments: FT nuclear-magnetic- 
resonance spectrometry, and FT ion-cyclotron-resonance spectrometry. Their technologies are unrelated to that of FTS ; the basic ideas and advantages are much the same [16].

Hence the FTS paradox: a technique wholly steeped in classical wave-optics, but understandable (and useful) only when scrutinized with photons.. With all due gratitude to Michelson, Rubens, and of course Fourier, the unveiling of FTS in the nineteen fifties has been the right idea at the right time.

\section{References}

[1] ConNES P., Spectrochem. Act. (1992, to be published).

[2] Proc. Int. Conf. Fourier Spectroscopy, Infr. Phys. 24 (1984).

[3] Connes P., Smyth M., Hadni A., GerbauX X., Opt. News 6 (March 1988).

[4] Kuhn H. G., Hartley Ch., Biog. Mem. Fell. Roy. Soc. 29 (1983) 269.

[5] JACQUINOT P., JOSA 44 (1954) 161.

[6] JACQuinot P., $17^{\mathrm{e}}$ Congrès GAMS (Paris, 1954) p. 25.

[7] PECK E., JOSA 45 (1955) 931.

[8] CONNES P., Rev. Opt. 35 (1956) 37.

[9] Colloque international sur les progrès récents en spectroscopie interférentielle, J. Phys. France 19 (1958).

[10] Gebbie H., Vanasse G., Nature 178 (1956) 432.

[11] Connes J., Gush H., J. Phys. France 20 (1959) $915 ; 21$ (1960) 645.

[12] Connes J., Rev. Opt. 40 (1961) 45116171231.

[13] Bouchareine P., Connes P., J. Phys. France 24 (1963) 134.

[14] Connes P., Michel G., Rev. Phys. Appl. 2 (1967) 175.

[15] PinaRd J., Thèse de $3^{\text {e }}$ Cycle, Université de Paris (1963).

[16] Connes P., Microchim. Acta (Wien) III (1987) 337. 